\title{
Morfologia radicular da dentição permanente de Sapajus apella: morfometria, anatomia macroscópica, ultraestrutura e propriedades físicas ${ }^{1}$
}

\author{
Luanna Melo Pereira Fernandes ${ }^{2}$, Elder Monteiro do Nascimento ${ }^{2}$, Camila Santa \\ Rosa Nunes ${ }^{2}$, Elma Pinto Vieira², Paula Dias Lins ${ }^{2}$, Francisco Bruno Teixeira ${ }^{2}$, \\ Roberta Souza D.A. Couto ${ }^{3}$ e Rafael Rodrigues Lima ${ }^{2 *}$
}

\begin{abstract}
Fernandes L.M.P., Nascimento E.M., Nunes C.S.R., Vieira E.P., Lins P.D., Teixeira F.B., Couto R.S.D.A. \& Lima R.R. 2014. [Root morphology of the permanent dentition Sapajus apella: morphometry, gross anatomy, ultrastructure and physical properties.] Morfologia radicular da dentição permanente de Sapajus apella: morfometria, anatomia macroscópica, ultraestrutura e propriedades físicas. Pesquisa Veterinária Brasileira 34(8):791-796. Laboratório de Biologia Estrutural e Funcional, Instituto de Ciências Biológicas, Universidade Federal do Pará, Rua Augsuto Correa 1, Guamá, Belém, PA 66075-900, Brazil. E-mail: rafalima@ufpa.br

This paper aimed the anatomical study including morphometric, ultrastructural and physical properties of permanent teeth of Sapajus apella primate. Ten adult male monkeys were studied. The teeth were evaluated for their length, root anatomy (external and internal considering the number of roots and canals), direction and shape of the root canal, density and diameter of the tubular root canal, dentin microhardness, and composition. The root anatomy showed specificity regarding the number of roots of the second premolar and the presence of the third premolar. We observed a decrease in the number and diameter of dentinal tubules along the root canal, a statistically significant difference when compared with the apical and cervical regions. Similarities with human teeth were found in regard to microhardness values and proportion of chemical elements in the root dentin.
\end{abstract}

INDEX TERMS: Dental anatomy, root morphology, primates, Cebus apella, Sapajus apella.

RESUMO.- 0 objetivo desse trabalho foi contribuir com o estudo anatômico, morfométrico, ultraestrutural e propriedades físicas dos dentes permanentes do primata $\mathrm{Sa}$ pajus apella. Para tal, foram utilizados 10 animais adultos e machos. Os dentes foram avaliados quanto ao seu comprimento e quanto à anatomia radicular externa e interna considerando número de raízes e canais, forma e direção radicular e forma do canal, assim como análise da densidade e diâmetro tubular do canal radicular, composição e microdureza dentinária. A anatomia radicular desse prima-

\footnotetext{
${ }^{1}$ Recebido em 8 de janeiro de 2014.

Aceito para publicação em 27 de julho de 2014

${ }^{2}$ Instituto de Ciências Biológicas, Universidade Federal do Pará (UFPA), Rua Augsuto Correa 1, Guamá, Belém, PA 66075-900, Brasil. *Autor para correspondência: rafalima@ufpa.br, rafaelrodrigueslima@hotmail.com

${ }^{3}$ Faculdade de Odontologia, UFPA, Rua Augusto Correa 1, Guamá, Belém, PA 66075-900.

${ }^{4}$ Laboratório de Biologia Estrutural e Funcional, Instituto de Ciências Biológicas, UFPA, Rua Augusto Correa 1, Guamá, Belém, PA 66075-900.
}

ta apresentou especificidades, como o número de raízes do segundo pré-molar superior e a presença do terceiro pré-molar. Quanto à densidade e o diâmetro dos túbulos dentinários, observou-se uma diminuição do número e diâmetro de túbulos ao longo do canal radicular, havendo diferença estatisticamente significante ao se comparar o terço apical com as regiões cervical e média, padrão de densidade e tamanho semelhantes a dentes humanos. Semelhanças também foram encontradas com dentes humanos quando comparados os valores de microdureza e proporção de elementos químicos encontrados na dentina radicular.

TERMOS DE INDEXAÇÃO: Anatomia dentária, morfologia radicular, primata, Cebus apella, Sapajus apella.

\section{INTRODUÇÃO}

o Cebus apella pertence ao filo Chordata, classe Mammalia, ordem Primates, família Cebidae e gênero Cebus sp. (Oppenheimer 1968), no entanto, este gênero tem sido considera- 
do um dos mais confusos grupos taxonômicos de mamíferos neotropicais (Alfaro et al. 2012a). De acordo com Silva Jr (2001) as 11 espécies de Cebus podem ser separados quanto à presença de tufo (subgênero Sapajus), com sete espécies denominadas de capuchinos robustos, ou ausência de tufo (subgênero Cebus), com quatro espécies denominadas de capuchinhos gracile. Esta separação taxonômica entre Sapajus e Cebus é amplamente discutida devido a variações ecológicas (Cunha et al. 2007, Vaz \& Port-Carvalho 2010), morfológicas (Wright et al. 2009), genéticas (Silva 2001, Amaral et al. 2008) e comportamentais (Alfaro et al. 2012a).

Dentre as espécies, se destaca Sapajus apella (Alfaro et al. 2012a, 2012b, Boubli et al. 2012), anteriormente Cebus apella. Sua distribuição biogeográfica abrange zonas neotropicais, especificamente na região do Bioma Amazônico (na Amazônia brasileira, peruana e boliviana), possuindo a maior distribuição geográfica de todos os Macacos do Novo Mundo (Hill 1962, Freese \& Oppenheimer 1981). Alojam-se preferencialmente em extratos arbóreos, porém, não apresentam grandes dificuldades adaptativas, sendo descritos em florestas primárias e secundárias, caatingas, mangues, palmeiras e campos (Silva Jr 2001, Boubli et al. 2012).

Estes Primatas não-humanos (PNH) são bastante sociáveis e encontrados formando extensos grupos compostos por 18 a 27 indivíduos, possuindo uma sobrevida em cativeiro que pode chegar até 20 ou 40 anos (Nowak \& Paradiso 1983). Devido a esta longevidade muito se tem estudado acerca desta espécie como aspectos de sua fisiologia reprodutiva (Nagle et al. 1980, Carosi \& Visalbergh 2002, Ortiz et al. 2005), parâmetros comportamentais (Carosi \& Visalbergh 2002, Pannozzo et al. 2007) e principalmente suas características morfológicas.

A literatura relata alguns estudos sobre a morfologia desta espécie em algumas regiões do sistema nervoso central (Guerreiro-Diniz et al. 2012, Phillips \& Thompson 2013), do sistema circulatório (Rosa et al. 2012) e de algumas estruturas ósseas (Byron 2009). Além disso, existem pesquisas sobre o aparelho estomatognático desta espécie que buscam associar a sua arquitetura morfológica com os seus hábitos comportamentais e sua dieta (Tylor \& Vinnyard 2009, Muchlinski et al. 2011).

Entretanto pouco se sabe sobre os aspectos da anatomia interna do canal radicular na dentição permanente deste primata neotropical e sua biologia oral. Desta forma este trabalho tem como objetivo contribuir com o estudo anatômico, morfométrico, ultraestrutural e propriedades físicas da dentição permanente do primata Sapajus apella colaborando assim para maiores dados específicos desta espécie, além de sua utilização como modelo para posteriores estudos em primatas neotropicais.

\section{MATERIAL E MÉTODOS}

Foram utilizados 10 animais da espécie Sapajus apella, machos, adultos, com idade estimada entre 10 e 12 anos de acordo com os dados de entrada dos animais no criadouro e média de massa

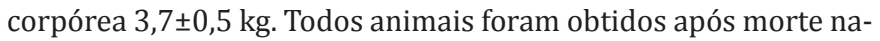
tural no Biotério da Universidade Federal do Pará. Este estudo foi aprovado pelo Comitê de ética em pesquisa com animais e experimentação da Universidade federal do Pará (BI0010-09).
Por não se ter planejado a data da morte dos animais, neste trabalho se utilizou apenas as estruturas mineralizadas dos dentes destes animais, já que o estado de conservação de qualquer tecido mole se apresentava comprometida para avaliação tecidual na coleta.

Os animais tiveram seus crânios dissecados, os dentes avulsionados de seus alvéolos com auxílio de instrumentais cirúrgicos e brocas esféricas acopladas ao motor de alta rotação, com intuito de preservação da integridade radicular. Após avulsão, os dentes foram limpos com curetas periodontais e os resíduos orgânicos aderidos à superfície radicular removidos, sendo armazenados em solução fisiológica até o momento do uso.

Análise anátomo-morfométrica. Os 360 dentes (80 incisivos, 40 caninos, 120 pré-molares, 120 molares) dos 10 animais foram avaliados quanto ao comprimento e quanto à anatomia radicular externa e interna, considerando número de raízes e canais por cada raiz, forma, direção radicular e forma do canal.

Para a análise do comprimento dentário utilizou-se o paquímetro Mundial Eberle Tools $(0,05 \times 150 \mathrm{~mm})$, mensurando os dentes em seus pontos mais extremos (Oliveira et al. 2006).

Para análise endodôntica, os dentes foram submetidos à tomadas radiográfica nos sentidos mésio-distal e vestíbulo-lingual, sendo para tanto usado o aparelho de raios-x Dabi Atlante spectro 70x de $8 \mathrm{~mA}$ (miliamperagem), com uma distância padronizada de $15 \mathrm{~cm}$, tempo de exposição de 0,3 segundos e filmes radiográficos (AGFA DENTUS M2 Speed E/F) com processamento efetuado em todos os filmes simultaneamente. Além disso, elementos de todos os grupos dentários foram submetidos ao desgaste longitudinal e a outra ao corte transversal. Os desgastes longitudinais foram realizados com auxílio de lixas d'água de gramatura de 1.000 e 1.500, para observar a trajetória do canal, iniciando o desgaste pela face mesial, para efeito de preservação da curvatura apical, até atingir o canal principal por inteiro, respeitando-se a curvatura do canal, caso existente. As secções transversais foram realizadas em três segmentos iguais, nos terços cervical, médio e apical das raízes para verificação da forma do canal. As amostras transversais e longitudinais tiveram suas imagens registradas em fotoestereomicroscópio e analisadas comparativamente.

Análise ultraestrutural por MEV. Para a análise ultraestrutural, alguns dentes foram clivados longitudinalmente. As hemi-secções de melhor integridade foram imersas em hipoclorito de sódio a $1 \%$ por 1 minuto para remover resíduos de material orgânico aderido à parede dentinária, sendo em seguida imersas em EDTA a $17 \%$ por 10 segundos, para remover detritos provenientes do seccionamento, passando por lavagem final de água destilada em banheira ultrassônica por 30 segundos, segundo protocolo estabelecido por Santana et al. (2013). Os dentes foram então desidratados em soluções crescentes de álcool, secos em temperatura ambiente, metalizados com platina e avaliados em microscópio eletrônico de varredura modelo LEO-1430 nas condições de análise para as imagens de elétrons secundários de: Corrente do Feixe de Elétrons $=90 \mu \mathrm{A}$; Voltagem de Aceleração Constante $=15 \mathrm{Kv}$ e Distância de Trabalho $=10 \mathrm{~mm}$.

Foram obtidas imagens nas magnificações de 1000X e 2000X do terço cervical, terço médio e terço apical da raiz, sendo na magnificação de 1000X mensurados a densidade de túbulos dentinários por área $\left(\mathrm{mm}^{2}\right)$ e na de $2000 \mathrm{X}$ mensurados o diâmetro médio dos túbulos dentinários $(\mu \mathrm{m})$ por terço radicular, utilizando para isso o software do próprio microscópio eletrônico de varredura. 0 número de túbulos dentinários em cada terço foi determinado pela contagem de todos os túbulos presentes e não obstruídos em cada terço radicular. Seus diâmetros foram determinados contando 10 túbulos dentinários em cada fotomicrografia obtida. Somente túbulos que apresentaram lúmen semelhante ao formato ao circular foram selecionados (Camargo, et al. 2007). 
Análise de microdureza da estrutura dentinária. Foram utilizados 2 dentes por animal, sendo caninos, onde foram seccionados no terço médio da raiz, obtendo-se amostras com dimensões de $1 \mathrm{~mm}$ x $1 \mathrm{~mm}$, com a face interna (parede do canal) voltadas para face de trabalho, as quais foram lixadas com auxílio de lixas de granulações crescentes (\#600, \#800 e \#1200) em uma politriz (modelo Aropol-e, SP, Brasil), com 500 rotações por minuto.

Em seguida as amostras foram lavadas em banheira ultra-sônica com água destilada por 1 minuto. As amostras foram incluídas em resina (Resina RQ-0100RF, São Paulo, Brasil) e polidas com pasta para polimento e disco de feltro (Diamond, FGM). A microdureza Knoop (KHN) foi determinada por um microdurômetro (Future Tech FM 700, Tóquio, Japão), onde foram realizadas 5 endentações por corpos de prova, espaçadas, aproximadamente, por $5 \mu \mathrm{m}$, com uma carga de $100 \mathrm{~g}$ e durante 10 segundos. As endentações foram realizadas paralelas ao longo eixo dos túbulos dentinários na superfície do canal radicular. Foram utilizados no total 20 corpos-de-prova, em todos foram realizadas 5 endentações, totalizando 100 endentações.

Análise da composição mineral. A microanálise por espectroscopia de energia dispersiva (EDS), utiliza um feixe focalizado de alta energia para gerar uma variedade de sinais na superfície das amostras. Os sinais que derivam de elétrons - interações da amostra revelam a informação sobre sua microestrutura para caracterizar a composição elementar de cada amostra. A EDS é uma técnica de microanálise química usada em conjunto com MEV.

A análise foi realizada por EDS (Oxford, Uedem, Alemanha) associada ao MEV Tabletop Microscope TM-3000 (Hitachi, Tóquio, Japão). Para tanto utilizamos 4 (quatro) dentes por animal de diferentes grupos de dentes (incisivos, caninos, pré-molares e molares).

Os dentes foram lavados em banheira ultra-sônica por 2 minutos e secos a temperatura ambiente. Foram retirados pequenos fragmentos de dentina da parede do canal de cada um dos dentes que foram montadas nos stubs, e submetidas à avaliação do EDS.
Análise estatística. Os valores encontrados na mensuração do comprimento dos dentes foram expressos com média \pm desvio padrão. A comparação estatística entre o terço radicular foram executados usando análise da variância de 1 via (ANOVA), as comparações múltiplas pós-teste foram executadas usando o teste de Tukey para densidade e diâmetro tubular. A análise de microdureza (Knoop) e composição mineral (EDS) foram expressos em média \pm desvio padrão, sendo feita apenas estatística descritiva. 0 nível de significância foi $\mathrm{p}<0.05$. Todos os testes foram executados usando o software estatístico (Graphpad Prisma 5.0, Graphpad Software, INC, USA).

\section{RESULTADOS}

$\mathrm{Na}$ análise da anatomia externa radicular, Sapajus apella apresentou na sua arcada superior e inferior um total de 36 dentes, sendo 4 ICS, 4 ICI, 4 ILS, 4 ILI, 6 PMS, 6 PMI, 6 MS e 6 MI, mostrando morfologia semelhante ao dos dentes humanos, diferindo apenas quanto ao número de raízes do 2oPMS (que apresentaram 2 raízes) e quanto a presença do 3 PM na arcada superior e inferior. 0 Quadro 1 mostra os dados referentes ao número de raízes e à anatomia radicular externa da dentição permanente de Sapajus apella.

Nos três terços radiculares, foi constatado a presença de variações no número de canais em cada raiz em elementos dentários do mesmo tipo. Os incisivos centrais e laterais superiores e o canino superior apresentaram semelhanças quanto à forma do canal, sendo circular nos terços cervical e médio e oval no terço apical. Os pré-molares e caninos inferiores assemelham-se somente no terço apical, ambos apresentando forma circular. 0 canino inferior apresenta forma riniforme nos terços cervical e médio, enquanto que os pré-molares inferiores se caracterizam por possuírem formato oval nesses terços. A descrição completa de todos

\section{Quadro 1. Anatomia radicular externa dos dentes de Sapajus apella}

\begin{tabular}{|c|c|c|c|}
\hline Dentes & $\begin{array}{l}\text { Número } \\
\text { de raízes }\end{array}$ & Forma & Direção \\
\hline $\begin{array}{l}\text { Incisivo central e } \\
\text { lateral superiores }\end{array}$ & Uma & $\begin{array}{l}\text { Cônica com achatamento mé- } \\
\text { sio-distal na porção apical }\end{array}$ & Reta com curvatura apical \\
\hline Canino Superior & Uma & Cônica e volumosa & $\begin{array}{l}\text { Curvatura gradual para palati- } \\
\text { na e distal }\end{array}$ \\
\hline $\begin{array}{l}1^{\circ}, 2^{\circ} \text { e } 3^{\circ} \text { Pré-molares } \\
\text { superiores }\end{array}$ & Duas & $\begin{array}{l}\text { Vestibular e palatina com } \\
\text { achatamento mésio-distal }\end{array}$ & Retas ou com curvatura apical \\
\hline $1^{\circ}$ Molar Superior & Três & $\begin{array}{l}\text { Mesiovestibular e distovesti- } \\
\text { bular são unidas,bifurcadas, } \\
\text { com achatamento mésio-dis- } \\
\text { tal. Raiz mesiovestibular mais } \\
\text { larga. A raiz palatina é cônica }\end{array}$ & $\begin{array}{l}\text { Mesiovestibular,distovestibular } \\
\text { e palatina são retas }\end{array}$ \\
\hline 2o Molar Superior & Duas & $\begin{array}{l}\text { Mesiovestibular e distovesti- } \\
\text { bular são unidas e cônicas. A } \\
\text { palatina é cônica }\end{array}$ & $\begin{array}{l}\text { Mesiovestibular,distovestibular } \\
\text { e palatina são retas }\end{array}$ \\
\hline 3o Molar Superior & Uma & Cônica e fusionada & Reta \\
\hline $\begin{array}{l}\text { Incisivo central e } \\
\text { lateral inferiores }\end{array}$ & Uma & $\begin{array}{l}\text { Cônica com achatamento mé- } \\
\text { sio-distal na porção apical }\end{array}$ & Reta \\
\hline Canino Inferior & Uma & Cônica e volumosa & $\begin{array}{l}\text { Curvatura gradual para palati- } \\
\text { na e distal }\end{array}$ \\
\hline $\begin{array}{l}1^{\circ}, 2^{\circ} \text { e } 3^{\circ} \text { Pré-molares } \\
\text { inferiores }\end{array}$ & Uma & $\begin{array}{l}\text { Achatamento mésio-distal e é } \\
\text { volumosa }\end{array}$ & Reta ou com curvatura apical \\
\hline 1o Molar Inferior & Duas & $\begin{array}{l}\text { Em ambas,achatamento mé- } \\
\text { sio-distal }\end{array}$ & Retas \\
\hline 2o Molar Inferior & Duas & $\begin{array}{l}\text { Achatamento mésio-distal e } \\
\text { bifurcação no terço apical,nas } \\
\text { duas }\end{array}$ & Retas \\
\hline 3o Molar Inferior & Uma & Cônica e fusionada & Reta \\
\hline
\end{tabular}


Quadro 2. Descrição anátomo-morfométrica e comprimento médio do dente e desvio-padrão $(\mathrm{mm})$ dos dentes selecionados de Sapajus apella

\begin{tabular}{|c|c|c|c|c|}
\hline Dentes & $\begin{array}{c}\text { Porção do } \\
\text { corte transversal }\end{array}$ & $\begin{array}{l}\text { Número } \\
\text { de canais }\end{array}$ & $\begin{array}{c}\text { Forma do } \\
\text { canal }\end{array}$ & $\begin{array}{l}\text { Comprimento Médio } \\
\text { Desvio-Padrão (mm) }\end{array}$ \\
\hline Incisivo & Terço cervical & Um & Circular & $12,4563 \pm 0,6062$ \\
\hline central & Terço médio & Um & Circular & \\
\hline superior & Terço apical & Um & Oval & \\
\hline Incisivo & Terço cervical & Um & Circular & $11,2688 \pm 0,4174$ \\
\hline lateral & Terço médio & Um & Circular & \\
\hline superior & Terço apical & Um & Oval & \\
\hline Canino & Terço cervical & Um & Circular & $21,1938 \pm 1,6003$ \\
\hline superior & Terço médio & Um & Circular & \\
\hline Terço apical & Um & Oval & & \\
\hline Canino & Terço cervical & Um & Riniforme & $20,0785 \pm 0,8827$ \\
\hline inferior & Terço médio & Um & Riniforme & \\
\hline Terço apical & Um & Circular & & \\
\hline $1^{\circ}, 2^{\circ}$ e $3^{\circ}$ & Terço cervical & Um & Oval & $11,1988 \pm 0,9888$ \\
\hline Pré-molares & Terço médio & Um & Oval & \\
\hline inferiores & Terço apical & Um & Circular & \\
\hline
\end{tabular}

os elementos encontra-se no Quadro 2, sendo descritos os achados mais frequentes.

Analisando-se a parede radicular, a média foi de 40.534 túbulos $/ \mathrm{mm}^{2}$. Ao se comparar a densidade tubular por regiões, observou-se uma diminuição do número de túbulos ao longo do canal radicular. A análise estatística mostrou haver diferença significante apenas em relação ao terço apical, onde as outras regiões avaliadas ao longo da parede interna da dentina apresentaram densidade tubular maior que este (Fig.1A-C). A análise estatística do diâmetro dos túbulos também mostrou diferenças ao se analisar o terço apical, o qual apresentou túbulos com diâmetro significativamente menor quando comparados aos terços cervical e médio (Fig.1D-F) (Quadro 3).

Os valores de microdureza pela avaliação da dureza Knoop revelaram um valor médio de $53,1 \mathrm{~kg} / \mathrm{mm}^{2}$ com desvio padrão de \pm 5 .0. Os demais resultados estão expressos no Quadro 4.
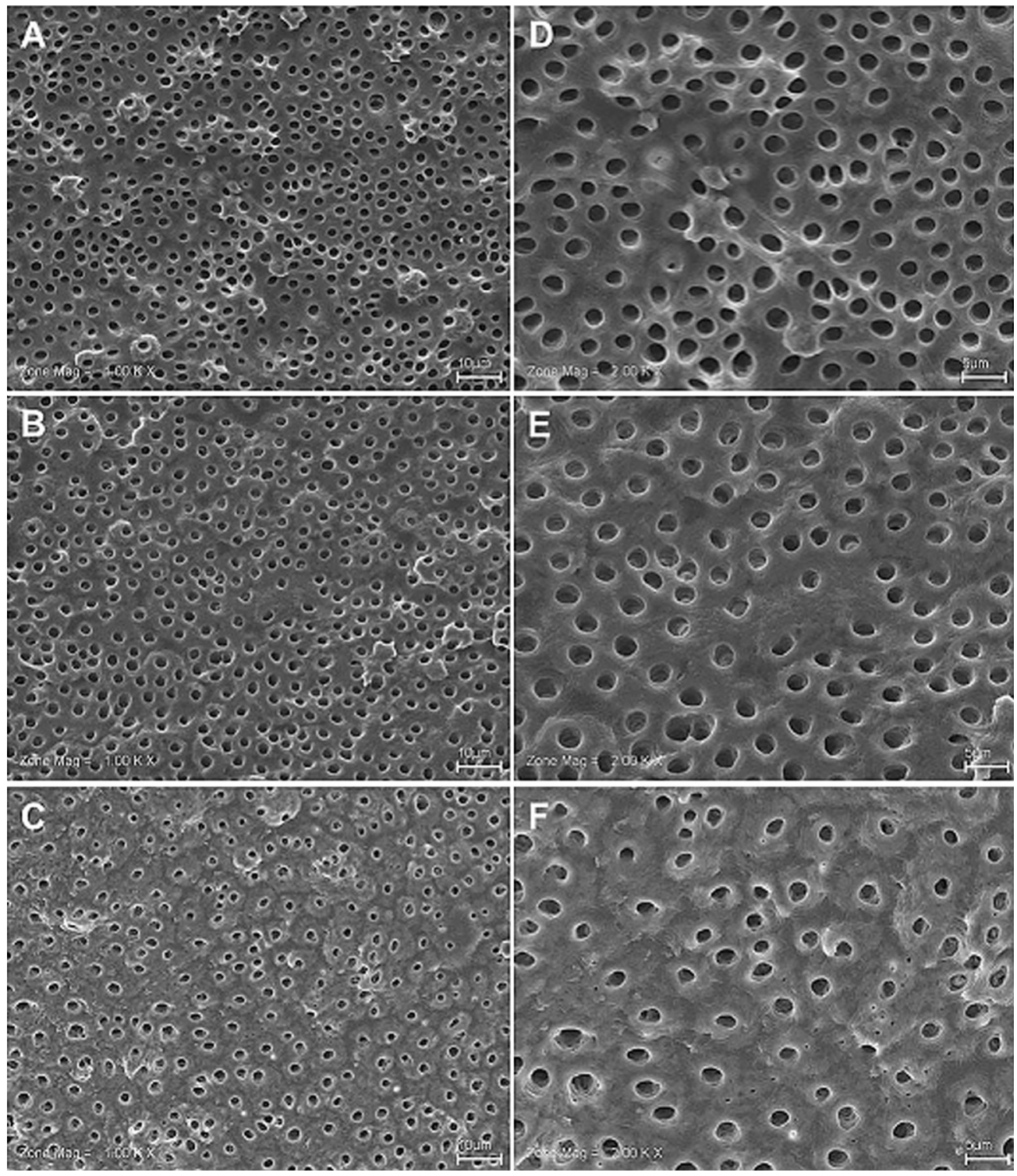

Fig.1. (A) A densidade dos túbulos dentinários nos terços cervical, (B) médio e (C) apical da raiz, magnificação de $1000 x$ e escala de $10 \mu \mathrm{m}$. (D) 0 diâmetro dos túbulos dentinários nos terços cervical, (E) médio e (F) apical da raiz, magnificação de 2000x e escala de $5 \mu \mathrm{m}$. 
Quadro 3. Número e diâmetro de túbulos por região analisada

\begin{tabular}{lcc}
\hline Região analisada & $\begin{array}{c}\text { Número de túbulos } \\
\left(\mathrm{mm}^{2}\right)\end{array}$ & $\begin{array}{c}\text { Diâmetro de túbulos } \\
(\mu \mathrm{m})\end{array}$ \\
\hline Terço cervical da raiz & 58.5000 & 2.1791 \\
Terço médio da raiz & 41.4000 & 2.2343 \\
Terço apical da raiz & $21.7000^{*}$ & $1.8040^{*}$
\end{tabular}

* Única região a apresentar diferença estatisticamente significante em relação as demais regiões da coluna na qual está inserido.

\section{Quadro 4. Representação dos valores médios de} microdureza

\begin{tabular}{lc}
\hline Tamanho da amostra & 20 \\
Mínimo & 48.0 \\
Máximo & 69.0 \\
Média aritmética & 53.1 \\
Desvio Padrão & 5.0 \\
Coeficiente de Variação & $9.36 \%$
\end{tabular}

Quadro 5. Representação dos valores médios dos elementos químicos presentes na dentina

\begin{tabular}{lcc}
\hline Elemento & Peso (\%) & Fórmula \\
\hline Sódio & 1.183 & $\mathrm{Na} 20$ \\
Magnésio & 1.513 & $\mathrm{MgO}$ \\
Fósforo & 20.428 & $\mathrm{P} 2 \mathrm{O5}$ \\
Cálcio & 34.292 & $\mathrm{CaO}$
\end{tabular}

Os valores médios dos elementos químicos presentes na dentina revelaram que o cálcio (34.29\%) e o fósforo $(20,42 \%)$ aparecem com uma maior porcentagem entre os outros elementos químicos. Os demais resultados estão expressos no Quadro 5.

\section{DISCUSSÃO}

Nesta investigação, pela primeira vez, se avaliou a estrutura do canal radicular da dentição permanente de Sapajus apella em suas propriedades físicas e características ultraestruturais e anatômicas. Para avaliação detalhada do padrão dentinário do canal radicular e a partir disso compararmos com o dente humano, realizamos um estudo de MEV para quantificar e qualificar a ultraestrutura dos dentes de Sapajus apella. Infelizmente, foi inviável a comparação da estrutura dentinária de primatas não-humanos com o animal estudado devido à escassez de estudos que avaliam todas as propriedades analisadas neste artigo.

Em nosso estudo, observamos que o canal radicular de um dente permanente de Sapajus apella é constituído internamente por dentina do tipo ortodentina. Segundo Baume (1980), a dentina do tipo ortodentina é composta de túbulos dentinários que se assemelham a pequenos tubos formados como resultado da mineralização da matriz dentinária ao redor dos prolongamentos odontoblásticos que podem atravessar toda a extensão da dentina, desde a polpa dentária até a junção amelo-dentinária ou à junção amelo-cementária. Este tipo de dentina pode ser encontrado em todos os mamíferos dentados, sendo o principal tipo constituinte da dentina humana (Baume 1980)

De acordo com os resultados de quantificação, a média de túbulos dentinários na parede interna da dentina de toda câmara pulpar e canal radicular foi de 43.750 túbulos/ $\mathrm{mm}^{2}$. Esta variável em comparação com a do dente humano evidenciou similaridade devido à literatura relatar a variação de valores entre 43.000 túbulos $/ \mathrm{mm}^{2}$ (Brajdić 2008) a 45.000 túbulos $/ \mathrm{mm}^{2}$ (Zavgorodniy 2008).

0 diâmetro médio dos túbulos dentinários em toda extensão da câmara pulpar e canal radicular encontrado na espécie estudada foi de $2,13 \mu \mathrm{m}$. Estudos em elementos dentários humanos revelam que o valor do diâmetro dos túbulos próximo à polpa está entre 2 a $3 \mu \mathrm{m}$ (Mjör 2009, Garberoglio 1976, Brajdić 2008, Zavgorodniy 2008), demonstrando novamente similaridade nos dentes destas espécies.

Em nosso estudo, também foi avaliada as propriedades físicas da dentina do canal radicular de Sapajus apella a partir da análise da microdureza. A dureza é uma propriedade mecânica que consiste na medida da resistência de um material a uma deformação plástica localizada. A mensuração da marca deixada pelo penetrador do microdurômetro é convertida para índices de dureza e expressa a propriedade do material em resistir à deformação plástica (Callister 2007). Atualmente, a ferramenta mais utilizada para estudar tecidos mineralizados é a Dureza Knoop (KHN).

Estudos evidenciam que a dentina humana possui microdureza aproximada de $50-60 \mathrm{Kg} / \mathrm{mm}^{2}$ (Banerjee 1999). Nosso estudo mostrou que a média da microdureza da dentina de Sapajus apella é de $53,1 \mathrm{~kg} / \mathrm{mm}^{2}$, mostrando-se equivalente aos valores encontrados da dentina nos dentes humanos segundo a literatura.

$\mathrm{Na}$ avaliação da composição química da dentina de Sapajus apella, nosso estudo utilizou a Espectroscopia de Energia Dispersiva de Raios X (EDS). Esta análise permite a identificação de elementos através de suas linhas características de raios X (Sánchez-Quevedo 1998). Foram analisadas as porcentagens de sódio, magnésio, fósforo e cálcio.

0 cálcio e o fósforo foram os elementos químicos com maior porcentagem na estrutura dentinária. Em nosso estudo encontramos os valores médios dos elementos químicos da dentina em que o cálcio correspondeu a 34,29\% e o fósforo a 20,42\% (Quadro 3) 0 valor médio de porcentagem observado em estudos da dentina de dentes permanentes humanos foi entre $26,7 \%$ e 50,77 para cálcio e $13,6 \%$ a $18,2 \%$ para o fósforo (Derise 1974, Oliveira et al. 2010). Diante disso, observamos mais uma vez similaridade de nossos resultados com os valores descritos na literatura para dentes humanos.

Diante de todo este exposto, este trabalho buscou realizar um estudo detalhado da dentina de Sapajus apella. A dentina é um tecido complexo que pode sofrer variações desde o início até o término de sua formação, devido a aspectos ambientais e patológicos (Baume 1980). 0 estudo detalhado da estrutura dentinária de animais permite obter achados sobre seu processo evolutivo e adaptativo durante os tempos e proporciona informações sobre seu estilo de vida e alimentação (Baume 1980, Santana et al. 2013).

A partir disso, concluímos que a dentina deste primata apresenta morfometria tubular semelhante à de humanos, microdureza equivalente e composição mineral similar ao tecido dentinário humano, tornando-se um modelo bio- 
lógico para utilização em posteriores estudos em primatas neotropicais e mesmo em estudos com aplicabilidade odontológica.

\section{REFERÊNCIAS}

Alfaro J.W.L., Silva Jr J.S. \& Rylands A.B. 2012a. How different are robust and gracile capuchin monkeys? An argument for the use of Sapajus and Cebus. Am. J. Primatol. 74:273-286.

Alfaro J.W.L., Matthews L., Boyette A.H., MacFarlan S.J,. Phillips K.A., Falótico T., Ottoni E., Verderane M., Izar P., Schulte M., Melin A., Fedigan L., Janson C. \& Alfaro M.E. 2012b. Anointing variation across wild capuchin populations: a review of material preferences, bout frequency and anointing sociality in Cebus and Sapajus. Am. J. Primatol. 74:299-314.

Amaral P.J.L., Finotelo F.M., De Oliveira E.H.C., Pissinatti A., Nagamachi C.Y. \& Pieczarka J.C. 2008. Phylogenetic studies of the genus Cebus (Cebidae: Primates) using chromosome painting and G-banding. BMC Evol. Biol. 8:169.

Banerjee A.M., Sherriff E., Kidd A. \& Watson T.F. 1999. A confocal microscopic study relating the autofluorescence of carious dentine to its microhardness. Brit. Dent. J. 187:206-210.

Baume L.J. 1980. The biology of pulcp and dentine (Monogr.). Oral Sci., Basel, 8:1-183.

Boubli J.P., Rylands A.B., Farias I.P., Alfaro M.E. \& Alfaro J.W.L. 2012. Cebus phylogenetic relationships: a preliminary reassessment of the diversity of the untufted capuchin monkeys. Am. J. Primatol. 74:381-393.

Brajdic D., Krznaric O.M., Azinovic Z., Mazan D. \& Baranovic M. 2008. Biological bases of dentin hybridization. Coll. Antropol. 32:901-906.

Byron C.D. 2009. Cranial suture morphology and its relationship to diet in Cebus. J. Hum. Evol. 57:649-655.

Camargo C.H.R., Siviero M., Camargo S.E.A., Oliveira S.H.G., Carvalho C.A.T. \& Valera M.C. 2007. Topographical, diametral, and quantitative analysis of dentin tubules in the root canals of human and bovine teeth. J. Endod. $33: 422-426$

Callister J.R. \& Rethwisch W.D. 2007. Mechanical properties of metals, p. 131-173. In: Materials Science and Engineering: an introduction. John Wiley and Sons, New York.

Carosi M. \& Visalberghi E. 2002. Analysis of tufted capuchin (Cebus apella) courtship and sexual behavior repertoire: changes throughout the female cycle and female interindividual differences. Am. J. Phys. Anthropol. 118:11-24.

Cunha F.A., Lopes M.A., Dantas S.M., Carmo N.A.S. \& Silva S.S.B. 2007. Registro de ocorrência de Cebus kaapori (Cebidae: Primates) na APA Lago de Tucuruí. Neotropical Primates 14:84-85.

Derise N.L., Ritchey S.J. \& Furr A.K. 1974. Mineral composition of normal human enamel and dentin and the relation of composition to dental caries. I. Macrominerals and comparison of methods of analyses. J. Dent. Res. 54:847-52.

Freese C.H. \& Oppenheimer J.R. 1981. The capuchin monkeys, genus $\mathrm{Ce}$ bus, p.331-389. In: Coimbra-Filho A.F. \& Mittermeier R.A. (Eds), Ecology and Behavior of Neotropical Primates. Vol.1. Academia Brasileira de Ciências, Rio de Janeiro.

Garberoglio R. \& Brannstrom M. 1976. Scanning electron microscopic investigation of human dentinal tubules. Arch. Oral Biol. 21:355-362.

Guerreiro-Diniz C., Paz R.M., Hamad M.H.S., Santos Filho C., Martins A.A.V., Neves H.B., Cunha E.D.S., Alves G.C., Sousa L.A., Dias I.A., Trévia N., Sousa A.A., Passos A., Lins N., Torres Neto J.B., Vasconcelos P.F.C. \& Picanço-Diniz C.W. 2010. Hippocampus and dentate gyrus of the $\mathrm{Ce}$ bus monkey: architectonic and stereological study. J. Chem. Neuroanat. 40:148-159.
Hill W.C. 1962. Primates: comparative anatomy and taxonomy. V. Cebidae Part B. Edinburgh University Press, Edinburgh, UK. 537p.

Mjor I.A. 2009. Dentin Permeability: the basis for understanding pulp reactions and adhesive technology. Braz. Dent. J. 20:3-16.

Muchlinski M.N., Docherty B.A., Alport L.J., Burrows A.M., Smith T.S. \& Paesani S.M. 2011. Behavioral and ecological consequences of sex-based differences in gustatory anatomy in Cebus apella. Anat. Rec. 294:21792192.

Nagle C.A., Riarte A., Quiroga S., Azorero R.M., Carril M., Denari J.H. \& Rosner J.M. 1980. Temporal relationship between follicular development, ovulation, and ovarian hormonal profile in the capuchin monkey (Cebus apella). Biol. Reprod. 23:629-635.

Nowak R.M. \& Paradiso J.L. 1983. Walker's Mammals of the World. $4^{\text {th }}$ ed. Johns Hopkins University Press, Baltimore. 1936p.

Oliveira M.A.H.M., Torres C.P., Gomes-Silva J.M., Chinelatti M.A., Menezes F.C.H., Palma-Dibb R.G. \& Borsatto M.C. 2010. Microstructure and mineral composition of dental enamel of permanent and deciduous teeth. Microsc. Res. Tech. 73:572-577.

Oppenheimer J.R. 1968. Behavior and ecology of the white faced monkey, Cebus capucinus, on Barro Colorado Island. PhD thesis, University of Illinois, USA. 179p.

Ortiz R.E., Ortiz A.C., Gajardo G., Zepeda A.J., Parraguez V.H., Ortiz M.E. \& Croxatto H.B. 2005. Cytologic, hormonal, and ultrasonographic correlates of the menstrual cycle of the New World monkey Cebus apella. Am. J. Primatol. 66:223-244.

Pannozzo P.L., Phillips K.A., Haas M.E. \& Mintz E.M. 2007. Social monitoring reflects dominance relationships in a small captive group of brown capuchin monkeys (Cebus apella), Ethology 113:881-888.

Phillips K.A. \& Thompson C.R. 2013. Hand preference for tool-use in capuchin monkeys (Cebus apella) is associated with asymmetry of the primary motor cortex. Am. J. Primatol. 75:435-440.

Rosa L., Silva Z., Pereira M., Santos L., Mitri F., Carvalho-Barros R. \& Silva D. 2012. Descriptive anatomic study of the great vessels of the heart in the capuchin monkey Cebus apella (Linnaeus, 1758). Anat. Histol. Embryol. 41:469-475

Sánchez-Quevedo M.C., Nieto-albano O.H., García J.M., Gómez de Ferraris M.E. \& Campos A. 1998. Electron probe microanalysis of permanent human enamel and dentine. A methodological and quantitative study. Histol. Histopathol. 13:109-113.

Santana L.N.S., Barbosa L.V.M., Teixeira F.B., Costa A.M.P., Fernandes L.M.P. \& Lima R.R. 2013. Morphology of the dentin structure of sloths Bradypus tridactylus: a light and scanning electron microscopy investigation. Anat. Histol. Embryol. Doi: 10.1111/ahe.12029. [Epub ahead of print]

Silva Jr J.S. 2001. Especiação nos macacos-prego e caiararas, gênero Cebus Erxleben, 1777 (Primates, Cebidae). Tese de Doutorado, Universidade Federal do Rio de Janeiro, Rio de Janeiro, RJ. 377p.

Taylor A.B. \& Vinyard C.J. 2009. Jaw-muscle fiber architecture in tufted capuchins favors generating relatively large muscle forces without compromising jaw gape. J. Hum. Evol. 57:710-720.

Vaz L.L. \& Port-Carvalho M. 2010. Ecologia e comportamento de um grupo de macacos-prego (Cebus spp.) (Primates: Cebidae) e frequência de contato com visitantes do Parque Estadual Alberto Löfgren. IF Sér. Reg., São Paulo, 42:121-125.

Wright B.W., Wright K.A., Chalk J., Verderane M.P., Fragaszy D., Visalberghi E., Izar P., Ottoni E.B., Constantino P. \& Vinyard C. 2009. Fallback foraging as a way of life: using dietary toughness to compare the fallback signal among capuchins and implications for interpreting morphological variation. Am. J. Phys. Anthropol. 140:687-699.

Zavgorodniy A.V., Rohanizadeh R. \& Swain M.V. 2008. Ultrastructure of dentine carious lesions. Arch. Oral Biol. 53:124-132. 